\title{
Conformal passivation of Multi-Channel GaN power transistors for reduced current collapse
}

\author{
Luca Nela, Student Member, Halil Kerim Yildirim, Catherine Erine, Student Member, Remco Van \\ Erp, Peng Xiang, Kai Cheng, and Elison Matioli, Member, IEEE
}

\begin{abstract}
Multi-channel power devices, in which several AlGaN/GaN layers are stacked to achieve multiple twodimensional electron gases (2DEGs), have recently led to a significant increase in the device conductivity while maintaining high breakdown voltage, resulting in excellent DC performances. However, their dynamic performance is yet to be demonstrated, especially due to the absence of an effective passivation technique for their 3D structure. Here, we present a surface passivation technology for multichannel devices based on a conformal deposition of a thin $\mathrm{SiO}_{2}$ interlayer followed by a low-pressure chemical vapor deposition (LPCVD) $\mathrm{Si}_{3} \mathrm{~N}_{4}$ layer around the multi-channel fins, which enables to effectively reduce the electron traps both at the AlGaN top surface and at the fin sidewalls. This approach led to a significant reduction of the dynamic onresistance $\left(\boldsymbol{R}_{\mathrm{ON}}\right)$ in multi-channel devices under large offstate voltages of $350 \mathrm{~V}$ and comparable dynamic performance with passivated single-channel reference devices. This work proves that, in addition to the excellent DC performance, the multi-channel technology can offer reduced current collapse, unveiling the potential of this platform for future power electronic applications.
\end{abstract}

Index Terms - Multi-channel, AlGaN/GaN, Tri-gate, HEMT, current collapse, $\mathrm{LPCVD} \mathrm{Si}_{3} \mathrm{~N}_{4}$, passivation

\section{INTRODUCTION}

$\mathrm{G}_{\mathrm{p}}$ aN-on-Si lateral devices have demonstrated outstanding potential for low- and medium-power conversion applications and a tremendous improvement in their performance has been achieved in recent years [1], [2]. Yet, the performance of current GaN High-Electron-Mobility Transistors (HEMTs) is still far below what the GaN material properties could offer [3]. In particular, the high-power figureof-merit for state-of-the-art $\mathrm{GaN}$ devices is still much lower with respect to the material limit, which translates into a larger

This work was supported in part by the Swiss National Science Foundation through Assistant Professor (AP) Energy under Grant PYAPP2_166901, in part by the European Research Council through the European Union's H2020 Program/ERC under Grant 679425, and in part by the ECSEL Joint Undertaking (JU) under grant agreement No 826392. The JU receives support from the European Union's Horizon 2020 research and innovation programme and Austria, Belgium, Germany, Italy, Norway, Slovakia, Spain, Sweden, Switzerland".L. Nela, H.K. Yildirim, C. Erine, R. Van Erp and E. Matioli are with the Power and Wide-band-gap Electronics Research Laboratory (POWERLAB), École Polytechnique Fédérale de Lausanne (EPFL), CH-1015 Lausanne, Switzerland (e-mail: luca.nela@epfl.ch; elison.matioli@epfl.ch). P. Xiang and K. Cheng are with Enkris Semiconductor, Inc., Suzhou 215123, China

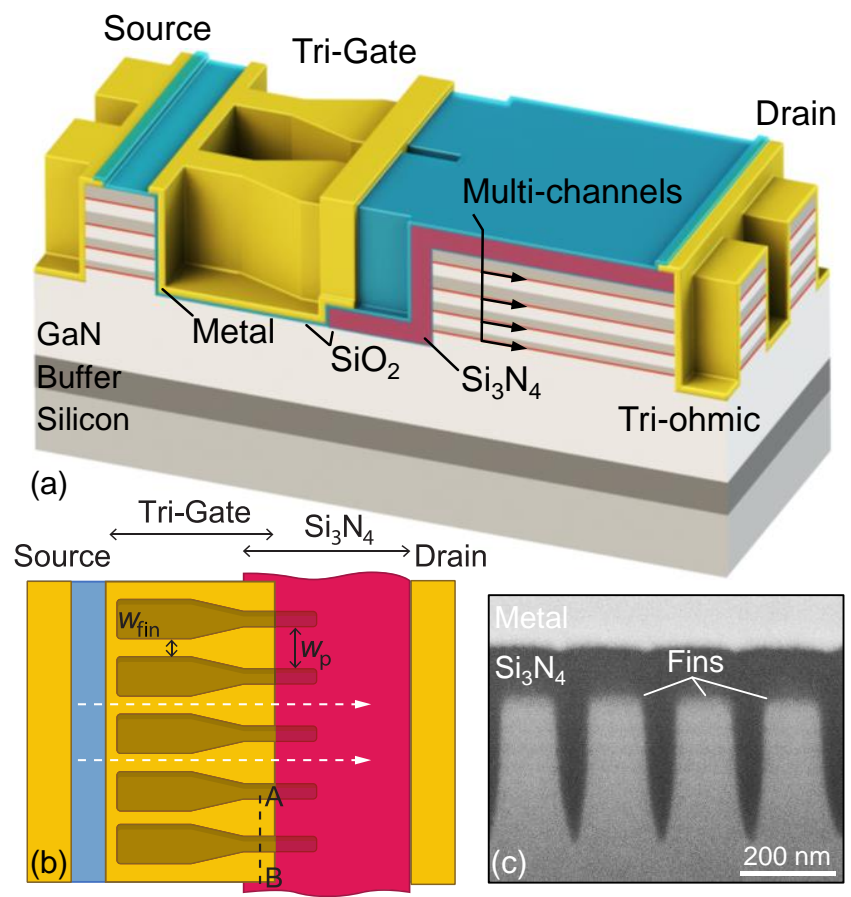

Fig. 1. (a) 3D schematics of the multi-channel tri-gate MOSHEMT with LPCVD $\mathrm{Si}_{3} \mathrm{~N}_{4}$ passivation layer. (b) Top-view schematics of the Tri-gate region indicating the fin dimensions. (c) Focused Ion Beam (FIB) crosssection along the $\mathrm{AB}$ line in Fig. 1(b) showing the passivated multi-channel fins conformably covered by the LPCVD $\mathrm{Si}_{3} \mathrm{~N}_{4}$ passivation layer.

specific on-resistance $\left(R_{\mathrm{ON}, \mathrm{SP}}\right)$ for a given breakdown voltage $\left(V_{\mathrm{BR}}\right)$.

Recently, multi-channel heterostructures, in which several $\mathrm{AlGaN} / \mathrm{GaN}$ layers are stacked to achieve multiple 2DEGs [4], [5], have been proposed to increase the heterostructure conductivity. While such structures were first proposed for RF applications [6]-[10], recent works have demonstrated multichannel devices for power applications [11]-[14], showing excellent DC performance and revealing the potential of the multi-channel platform for power conversion applications. In particular, a reduction of about 3 times in $R_{\mathrm{ON}, \mathrm{SP}}$ with respect to conventional single-channel devices was achieved while maintaining similar $V_{\mathrm{BR}}$ [13].

Despite the encouraging perspectives, previous works have focused solely on the DC characterization of multi-channel devices while their performance during switching operation is yet to be demonstrated. $\mathrm{AlGaN} / \mathrm{GaN}$ power devices suffer from severe current collapse during high-voltage switching mainly due to electron trapping by surface trapping states. For conventional single-channel devices, this is typically addressed by the deposition of a passivation layer to eliminate the trapping sites at the top AlGaN surface [15]-[17].

In multichannel structures, it has been shown that tri-gate-based 

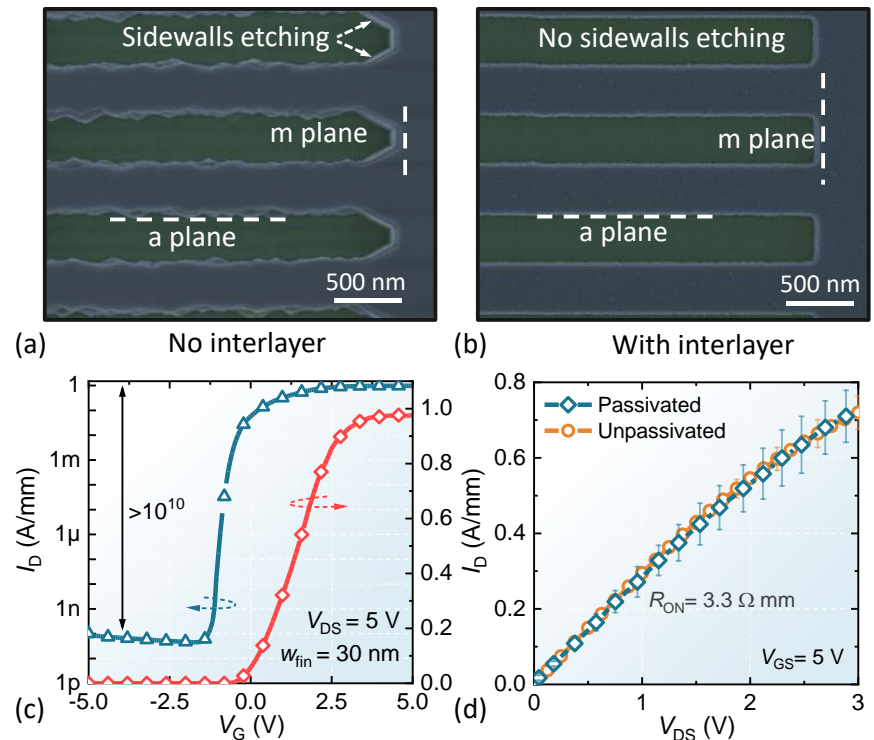

(b)

With interlayer

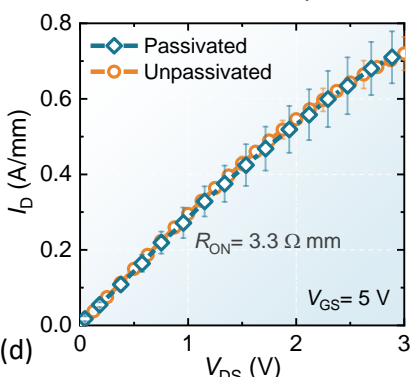

Fig. 2. (a) SEM image of multi-channel fins after the $100 \mathrm{~nm} \mathrm{LPCVD} \mathrm{Si}_{3} \mathrm{~N}_{4}$ deposition without and with (b) a $2 \mathrm{~nm}$-thick $\mathrm{SiO}_{2}$ protective interlayer. (c) DC transfer curve of the passivated device after the $\mathrm{Si}_{3} \mathrm{~N}_{4}$ etching in the Trigate region. $W_{\mathrm{p}}$ was set to $100 \mathrm{~nm}$ (d) DC output curve of multi-channel devices with and without the passivation layer showing similar $\mathrm{R}_{\mathrm{ON}}$.

field-plate structures [13], [18] are required to manage the large off-state electric fields (Fig. 1 (a-b)). Such architecture demands effective passivation of both the top $\mathrm{AlGaN}$ surface and the fins sidewalls, which combined with the presence of multiple 2DEGs, poses additional challenges for the passivation of multi-channel devices. This leaves unanswered the important question of whether multi-channel devices can be effectively passivated and whether conventional surface passivation techniques can be applied to such devices.

In this work, we present a surface passivation technology for multi-channel devices based on a conformal $\mathrm{SiO}_{2} / \mathrm{Si}_{3} \mathrm{~N}_{4}$ layer around the multi-channel fins, which enables to effectively reduce the electron traps both at the AlGaN top surface and at the fin sidewalls. Thanks to this approach, we demonstrate that multi-channel devices can be effectively passivated, offering reduced current collapse at high-voltage operation, which represents a key advance on the development of the multichannel technologies for power applications.

\section{DEVICE FABRICATION}

The multi-channel AlGaN/GaN heterostructure included 4 parallel 2DEG channels. The top 3 channels were composed of $20 \mathrm{~nm} \mathrm{Si}$-doped AlGaN barrier (with Si concentration of $10^{19}$ $\left.\mathrm{cm}^{-3}\right), 1 \mathrm{~nm}$ AlN spacer, and a $20 \mathrm{~nm} \mathrm{GaN}$ channel layer, while the last channel consisted of $10 \mathrm{~nm} \mathrm{AlGaN}$ barrier with $5 \times 10^{18}$ $\mathrm{cm}^{-3} \mathrm{Si}$ doping, $1 \mathrm{~nm}$ AlN spacer and $20 \mathrm{~nm} \mathrm{GaN}$ channel. More details on the design of such heterostructure can be found in Ref. [13]. The Hall mobility and carrier concentration of the multi-channel structure were $1930 \mathrm{~cm}^{2} \cdot \mathrm{V}^{-1} \mathrm{~s}^{-1}$ and $3.9 \times 10^{13}$ $\mathrm{cm}^{-2}$ respectively, resulting in a sheet resistance of $83 \Omega / \mathrm{sq}$.

The fabrication started with electron-beam lithography to define the device mesa and the tri-gate structures in the gate region (Fig. 1 (a-c)). The sample was then etched by $\mathrm{Cl}_{2}$-based inductively coupled plasma etching (ICP) to a depth of $280 \mathrm{~nm}$. The design of the tri-gate region is shown in Fig. 1 (b) and is similar to the one described in Ref. [13]. An $\mathrm{O}_{2}$ plasma/ $\mathrm{HCl}$ cycled treatment was performed to minimize etching damages

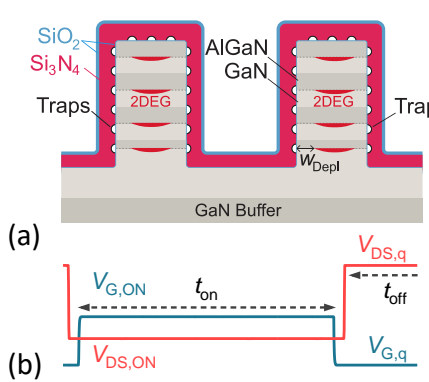

(b)
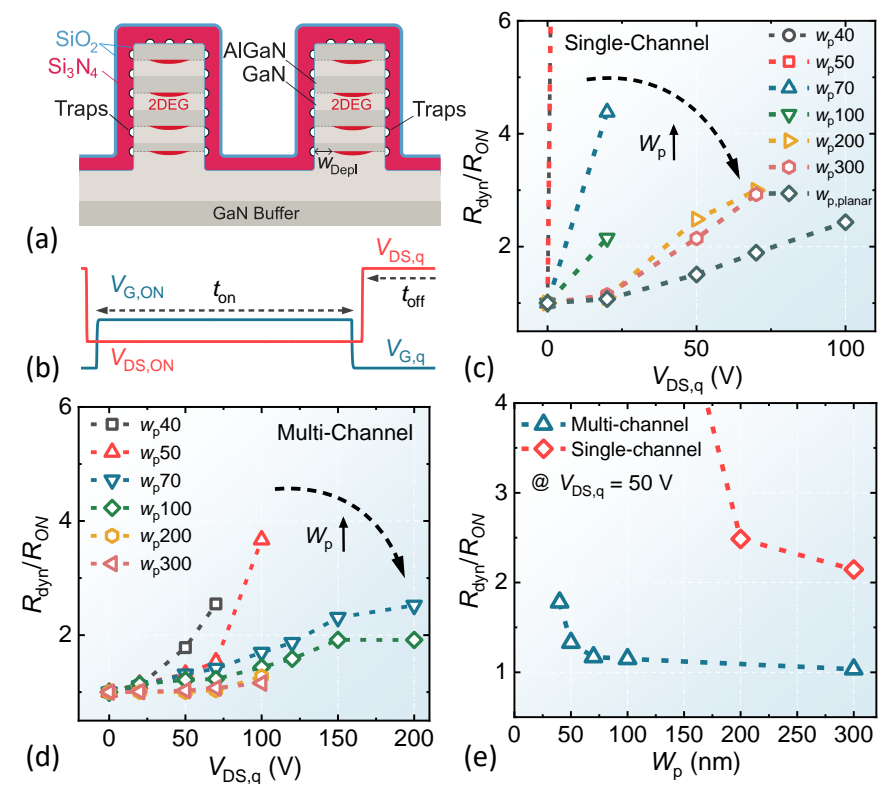

Fig. 3. (a) Cross-section illustration of the passivated multi-channel fins highlighting the presence of sidewalls traps. (b) Schematics of the $R_{\mathrm{ON}, \mathrm{dyn}}$ measurement. (c) Normalized $R_{\mathrm{dyn}}$ for the single-channel reference devices and the multi-channel devices (d) as a function of $V_{\mathrm{DS}, \mathrm{q}}$ for different tri-gate termination widths $w_{\mathrm{p}}$. $w_{\mathrm{p} \text {,planar }}$ stands for the conventional termination for single-channel devices on the planar region. $w_{\text {fin }}$ was set to $30 \mathrm{~nm}$. (e) Normalized $R_{\mathrm{dyn}}$ comparison as a function of $w_{\mathrm{p}}$ for single- and multi-channel devices at $V_{\mathrm{DS}, \mathrm{q}}$ of $50 \mathrm{~V}$.

on the fins sidewalls, followed by RCA cleaning of the sample. A $100 \mathrm{~nm}$-thick LPCVD $\mathrm{Si}_{3} \mathrm{~N}_{4}$ passivation layer was deposited at $770^{\circ} \mathrm{C}$ with a flow of $30 \mathrm{sccm}$ of $\mathrm{SiH}_{2} \mathrm{Cl}_{2}$ and $180 \mathrm{sccm}$ of $\mathrm{NH}_{3}$ at a chamber pressure of $100 \mathrm{mT}$.

After the LPCVD deposition, fin structures aligned along the m-direction showed a strong crystallographic etching which caused significant damages to their sidewalls (Fig. 2 (a)) and even their complete removal for widths below $300 \mathrm{~nm}$. No significant etching was instead observed for the $\mathrm{m}$ - and cplanes. This issue was possibly due to $\mathrm{GaN}$ surface desorption during the LPCVD process [19], [20], and was successfully resolved by the introduction of a $2 \mathrm{~nm}$-thick $\mathrm{SiO}_{2}$ interlayer. The $\mathrm{SiO}_{2}$ was deposited by atomic layer deposition (ALD) before the LPCVD deposition and effectively protected the surface, resulting in no etching of the tri-gate sidewalls (Fig. 2 (b)). This was particularly important since the slanted portions of the fins would be completely deformed after the LPCVD $\mathrm{Si}_{3} \mathrm{~N}_{4}$ deposition.

The LPCVD $\mathrm{Si}_{3} \mathrm{~N}_{4}$ was removed in the gate and contact regions with low power $(30 \mathrm{~W}) \mathrm{RIE}$ etching. Since the $100 \mathrm{~nm}$-thick $\mathrm{Si}_{3} \mathrm{~N}_{4}$ filled the trenches between the fins (Fig. 1 (c)), about 150 $\mathrm{nm}$ of over-etching was performed to remove the passivation layer and enable the formation of the tri-gate structure around all of the embedded channels. Ohmic contacts were formed by a Ti/Al/Ti/Ni/Au metal stack, and annealed at $780^{\circ} \mathrm{C}$ for $30 \mathrm{~s}$. A $25 \mathrm{~nm}$-thick layer of $\mathrm{SiO}_{2}$ was deposited by ALD at $300^{\circ} \mathrm{C}$ and served as the gate oxide, followed by a Pt/Au $(40 \mathrm{~nm} / 100$ $\mathrm{nm})$ gate metal stack. The device dimensions are $\mathrm{L}_{\mathrm{GS}}=1 \mu \mathrm{m}$, $\mathrm{L}_{\mathrm{G}}=1.5 \mu \mathrm{m}$ and $\mathrm{L}_{\mathrm{GD}}=10 \mu \mathrm{m}$.

Reference single-channel devices were co-fabricated in the same batch undergoing the same fabrication process. $w_{\text {fin }}$ was set to $30 \mathrm{~nm}$ throughout the paper since, while beneficial to tune the device threshold voltage, it showed no impact on the dynamic on-resistance. All quantities reported in the 

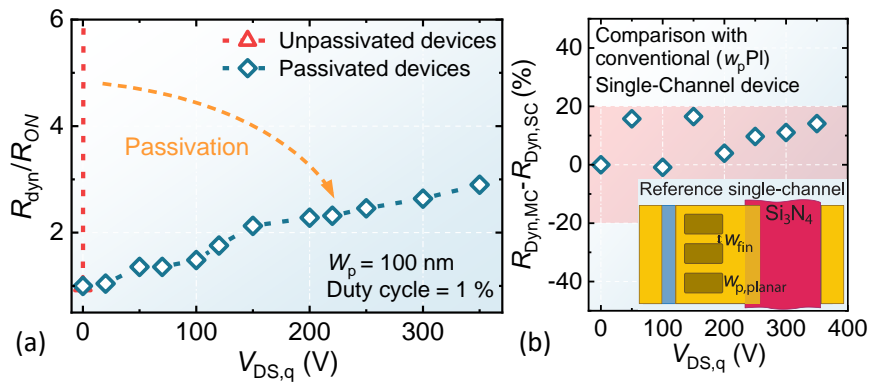

Fig. 4. (a) Normalized $R_{\text {dyn }}$ comparison for the multi-channel devices with and without the LPCVD $\mathrm{Si}_{3} \mathrm{~N}_{4}$ passivation layer. (b) Difference (normalized by the average) between normalized $R_{\mathrm{dyn}}$ for multi- and single-channel devices. Single-channel devices with conventional planar gate termination ( $\left.w_{\mathrm{p}, \text { planar }}\right)$ were considered for the comparison (bottom right inset).

manuscript have been normalized by the total device width $(W$ $=60 \mu \mathrm{m})$.

\section{DEVICE CHARACTERIZATION}

The transfer characteristic of a multi-channel tri-gate device having fin width ( $w_{\text {fin }}$ ) of $30 \mathrm{~nm}$ is shown in Fig. 2 (c). The $\mathrm{Si}_{3} \mathrm{~N}_{4}$ removal in the trenches between fins allowed to form the trigate structure around all of the embedded channels. This leads to excellent control over the multi-channel structure, resulting in high $I_{\mathrm{ON}} / I_{\mathrm{OFF}}$ above $10^{10}$. Thanks to the optimized etching recipe and the large carrier density of the multi-channel heterostructure, the $\mathrm{Si}_{3} \mathrm{~N}_{4}$ over-etching in the trench region did not significantly affect the device output curve. A very low DC on-resistance $\left(R_{\mathrm{ON}}\right)$ of $3.3 \Omega \cdot \mathrm{mm}$ was measured, which corresponds to a specific on-resistance $\left(R_{\mathrm{ON}, \mathrm{sp}}\right)$ of $0.51 \mathrm{~m} \Omega \cdot \mathrm{cm}^{2}$. This value is very similar to the one from reference unpassivated devices (Fig. 2 (d)), confirming no noticeable degradation as a result of the $\mathrm{Si}_{3} \mathrm{~N}_{4}$ removal.

To effectively manage the high off-state field in such conducting heterostructures, multi-channel devices require trigate-based field-plate solutions with the gate electrode terminating in the fin region rather than on the planar portion [13] (Fig. 1 (a-b)). This leads to the exposure of new crystal surfaces after the fin etching and damages during the dry etching process, potentially resulting in additional trapping sites on the fin sidewalls (Fig. 3 (a)). To investigate the effect of sidewalls traps on the dynamic behavior, devices with different tri-gate termination width $w_{\mathrm{p}}$ (see Fig. 1 (a)) were fabricated both on single- and multi-channel heterostructures and their dynamic on-resistance $\left(R_{\mathrm{dyn}}\right)$ was extracted by pulsed I-V characteristics (Fig. 3 (b)). The device was stressed in the OFF state at quiescent drain voltage $\left(V_{\mathrm{DS}, \mathrm{q}}\right)$ for a time $\left(t_{\mathrm{off}}\right)$ and then suddenly turned $\mathrm{ON}$ for a short time $t_{\mathrm{on}}$ during which the output curve was measured. For this measurement $t_{\text {on }}$ was set to $50 \mu \mathrm{s}$ and $t_{\text {off }}$ to $5 \mathrm{~ms}$, resulting in a duty cycle of $1 \%$. In the OFF state, the quiescent gate voltage $\left(V_{\mathrm{G}, \mathrm{q}}\right)$ was $-3 \mathrm{~V}$ while the quiescent drain voltage $\left(V_{\mathrm{DS}, \mathrm{q}}\right)$ was varied. In the $\mathrm{ON}$ state, $V_{\mathrm{G}}$ was $5 \mathrm{~V}$ and $V_{\mathrm{DS}}$ was swept from $0 \mathrm{~V}$ to $3 \mathrm{~V}$.

Fig. 3 (c) shows the normalized $R_{\mathrm{dyn}}$ as a function of $V_{\mathrm{DS}, \mathrm{q}}$ at different $w_{\mathrm{p}}$ for the reference single-channel devices. Despite the passivation layer, devices with small $w_{\mathrm{p}}$ show highly degraded dynamic performance, which drastically improved as $w_{\mathrm{p}}$ increases. Such behavior confirms the presence of sidewalls traps, whose effect increases significantly as the fin termination width is reduced, degrading $R_{\text {dyn }}$. While a similar trend of $R_{\text {dyn }}$ with $w_{\mathrm{p}}$ is observed for multi-channel devices (Fig. 3 (d)), its increase is lower with respect to the single-channel case and becomes considerable only for small $w_{\mathrm{p}}<50 \mathrm{~nm}$. Such an effect can be explained by the reduced effectiveness of the trapped electrons, acting as a virtual gate [21], in depleting the multichannel fins. This is due to the much higher carrier density in the multi-channel heterostructure (almost $4 \mathrm{x}$ larger than the single-channel case) and to the weak influence exerted by the top $\mathrm{AlGaN}$ surface traps on the buried channels. Similarly to the channel control by the tri-gate structure for which, at a given fin width, a larger gate voltage is required to turn-off a multichannel device [13], trapped electrons acting as virtual gate are much less effective in depleting a multi-channel fin rather than a single-channel one. For this reason, much smaller values of $w_{\mathrm{p}}$ are required to have a good dynamic performance for multichannel devices, which is shown in Fig. 3 (e) where $R_{\text {dyn }}$ as a function of $w_{\mathrm{p}}$ is compared for single- and multi-channel devices at a fixed $V_{\mathrm{DS}, \mathrm{q}}$. While multi-channel devices present quite constant $R_{\text {dyn }}$ for $w_{\mathrm{p}}$ above $70 \mathrm{~nm}$, single-channel devices require $w_{\mathrm{p}}$ of 200-300 $\mathrm{nm}$ to obtain a reasonable but still much higher $R_{\text {dyn }}$ than for multi-channels. Most importantly, effective multi-channel device passivation can be achieved in the $w_{\mathrm{p}}$ range between $70-150 \mathrm{~nm}$ (Fig. 3 (e)), which is the range required to achieve proper electric field management, as larger $w_{\mathrm{p}}$ designs result in degradation of the device blocking capability [13].

The dynamic behavior of multi-channel devices with and without the passivation layer is shown in Fig. 4 (a). While unpassivated devices present almost no current for $V_{\mathrm{DS}, \mathrm{q}}$ as low as $20 \mathrm{~V}$, passivated devices show reduced $R_{\text {dyn }}$ up to $V_{\mathrm{DS}, \mathrm{q}}$ of 350 $\mathrm{V}$, demonstrating that effective passivation at high operating voltage can be achieved for multi-channel devices. Notably, their dynamic performance is comparable to the one of reference single-channel MOSHEMTs with a planar gate termination, which is the conventional and optimal architecture for single-channel devices (Fig. 4 (b)). This indicates that the presence of traps on the multi-channel fin sidewalls can be effectively suppressed and that good dynamic performance, comparable to the single-channel devices for a given passivation technique, can be achieved for multi-channel MOSHEMTs. Further improvements are possible by tuning the etching process to reduce the damages to the fin sidewalls and by optimizing the deposition of the protective interlayer and LPCVD $\mathrm{Si}_{3} \mathrm{~N}_{4}$.

\section{CONCLUSIONS}

In this work, we presented a surface passivation technology for multi-channel devices based on a conformal $\mathrm{SiO}_{2} / \mathrm{Si}_{3} \mathrm{~N}_{4}$ passivation layer around the multi-channel fins. Thanks to this approach, multi-channel MOSHEMTs with significantly reduced dynamic $R_{\mathrm{ON}}$ up to large charging voltages of $350 \mathrm{~V}$ and comparable dynamic performance with respect to singlechannel reference devices were achieved. These results prove that the multi-channel devices can present excellent DC and dynamic performances, showing the potential of this technology for future power devices. 


\section{REFERENCES}

[1] K. J. Chen, O. Haberlen, A. Lidow, C. L. Tsai, T. Ueda, Y. Uemoto, and $\mathrm{Y}$. Wu, "GaN-on-Si power technology: Devices and applications," IEEE Trans. Electron Devices, vol. 64, no. 3, pp. 779795, 2017, doi: 10.1109/TED.2017.2657579.

[2] H. Amano, Y. Baines, E. Beam, M. Borga, T. Bouchet, R. Chu, C. De Santi, and M. M. De Souza, "The $2018 \mathrm{GaN}$ power electronics roadmap," J. Phys. D. Appl. Phys., vol. 51, p. 163001, 2018.

[3] B. J. Baliga, "Gallium nitride devices for power electronic applications," Semicond. Sci. Technol., vol. 28, no. 7, 2013, doi: 10.1088/0268-1242/28/7/074011.

[4] Y. Cao, K. Wang, G. Li, T. Kosel, H. Xing, and D. Jena, "MBE growth of high conductivity single and multiple AlN/GaN heterojunctions," J. Cryst. Growth, vol. 323, no. 1, pp. 529-533, 2011, doi: 10.1016/j.jcrysgro.2010.12.047.

[5] S. Heikman, S. Keller, D. S. Green, S. P. DenBaars, and U. K. Mishra, "High conductivity modulation doped $\mathrm{AlGaN} / \mathrm{GaN}$ multiple channel heterostructures," J. Appl. Phys., vol. 94, no. 8, pp. 5321-5325, 2003 , doi: $10.1063 / 1.1610244$.

[6] R. S. Howell, E. J. Stewart, R. Freitag, J. Parke, B. Nechay, H. Cramer, M. King, S. Gupta, J. Hartman, M. Snook, I. Wathuthanthri, P. Ralston, K. Renaldo, H. G. Henry, and R. C. Clarke, "The SuperLattice Castellated Field Effect Transistor (SLCFET): A novel high performance Transistor topology ideal for RF switching," Int. Electron Devices Meet. IEDM, no. February, pp. 11.5.1-11.5.4, 2014, doi: 10.1109/IEDM.2014.7047033.

[7] K. Shinohara, C. King, E. J. Regan, J. Bergman, A. D. Carter, A. Arias, M. Urteaga, B. Brar, R. Page, R. Chaudhuri, M. Islam, H. Xing, and D. Jena, "GaN-Based Multi-Channel Transistors with Lateral Gate for Linear and Efficient Millimeter-Wave Power Amplifiers," IEEE MTT-S Int. Microw. Symp. Dig., pp. 1133-1135, 2019, doi: 10.1109/mwsym.2019.8700845.

[8] J. Chang, S. Afroz, K. Nagamatsu, K. Frey, S. Saluru, J. Merkel, S. Taylor, E. Stewart, S. Gupta, and R. Howell, "The super-lattice castellated field-effect transistor: A high-power, high-performance RF amplifier," IEEE Electron Device Lett., vol. 40, no. 7, pp. 10481051, 2019, doi: 10.1109/LED.2019.2917285.

[9] R. Chu and K. Shinohara, III-Nitride Electronic Devices. Elsevier, 2019.

[10] T. Palacios, A. Chini, D. Buttari, S. Heikman, A. Chakraborty, S. Keller, S. P. DenBaars, and U. K. Mishra, "Use of double-channel heterostructures to improve the access resistance and linearity in GaN-based HEMTs," IEEE Trans. Electron Devices, vol. 53, no. 3, pp. 562-565, 2006, doi: 10.1109/TED.2005.863767.

[11] J. Ma, G. Kampitsis, P. Xiang, K. Cheng, and E. Matioli, "MultiChannel Tri-gate GaN Power Schottky Diodes with Low ONResistance," IEEE Electron Device Lett., vol. 40, no. 2, pp. 275-278, 2018, doi: 10.1109/LED.2018.2887199.

[12] J. Ma, C. Erine, P. Xiang, K. Cheng, and E. Matioli, "Multi-channel tri-gate normally-on / off AlGaN / GaN MOSHEMTs on Si substrate with high breakdown voltage and low ON-resistance," Appl. Phys. Lett., vol. 242102, pp. 1-5, 2018, doi: 10.1063/1.5064407.

[13] J. Ma, C. Erine, M. Zhu, L. Nela, P. Xiang, K. Cheng, and E. Matioli, "1200 V Multi-Channel Power Devices with $2.8 \Omega \cdot \mathrm{mm}$ ONResistance," 2019 IEEE Int. Electron Devices Meet., 2019, doi: 10.1109/IEDM19573.2019.8993536.

[14] M. Xiao, Y. Ma, K. Cheng, K. Liu, A. Xie, E. Beam, Y. Cao, and Y. Zhang, "3.3 kV Multi-Channel AlGaN/GaN Schottky Barrier Diodes With P-GaN Termination," IEEE Electron Device Lett., vol. 41, no. 8, pp. 1177-1180, 2020, doi: 10.1109/LED.2020.3005934.

[15] Z. Zhang, G. Yu, X. Zhang, X. Deng, S. Li, Y. Fan, S. Sun, L. Song, S. Tan, D. Wu, W. Li, W. Huang, K. Fu, Y. Cai, Q. Sun, and B. Zhang, "Studies on high-voltage GaN-on-Si MIS-HEMTs using LPCVD Si3N4as gate dielectric and passivation layer," IEEE Trans. Electron Devices, vol. 63, no. 2, pp. 731-738, 2016, doi: 10.1109/TED.2015.2510445.

[16] M. Hua, C. Liu, S. Yang, S. Liu, K. Fu, Z. Dong, Y. Cai, B. Zhang, and K. J. Chen, "GaN-based metal-insulator-semiconductor highelectron-mobility transistors using low-pressure chemical vapor deposition SiNx as gate dielectric," IEEE Electron Device Lett., vol. 36, no. 5, pp. 448-450, 2015, doi: 10.1109/LED.2015.2409878.

[17] W. M. Waller, M. Gajda, S. Pandey, J. J. T. M. Donkers, D. Calton, J. Croon, J. Sonsky, M. J. Uren, and M. Kuball, "Control of Buffer-
Induced Current Collapse in AlGaN/GaN HEMTs Using SiNx Deposition," IEEE Trans. Electron Devices, vol. 64, no. 10, pp. 4044-4049, 2017, doi: 10.1109/TED.2017.2738669.

[18] J. Ma and E. Matioli, "Slanted Tri-Gates for High-Voltage GaN Power Devices," IEEE Electron Device Lett., vol. 38, no. 9, pp. 13051308, 2017, doi: 10.1109/LED.2017.2731799.

[19] M. Hua, S. Member, J. Wei, G. Tang, Z. Zhang, Q. Qian, X. Cai, N. Wang, and K. J. Chen, "Normally-Off LPCVD-SiNx/GaN MIS-FET With Crystalline Oxidation Interlayer," IEEE Electron Device Lett., vol. 38, no. 7, pp. 929-932, 2017, doi: 10.1109/LED.2017.2707473.

[20] M. Hua, Z. Zhang, J. Wei, J. Lei, G. Tang, K. Fu, Y. Cai, B. Zhang, and K. J. Chen, "Integration of LPCVD-SiNx gate dielectric with recessed-gate E-mode GaN MIS-FETs: Toward high performance, high stability and long TDDB lifetime," Int. Electron Devices Meet. IEDM, pp. 10.4.1-10.4.4, 2017, doi: 10.1109/IEDM.2016.7838388.

[21] R. Vetury, N. Q. Zhang, S. Keller, and U. K. Misha, "The impact of surface states on the DC and RF characteristics of $\mathrm{AlGaN} / \mathrm{GaN}$ HFETs," IEEE Trans. Electron Devices, vol. 48, no. 3, pp. 560-566, 2001, doi: 10.1109/16.906451. 